\title{
Experimental feasibility of molecular two-photon absorption with isolated time-frequency-entangled photon pairs
}

\author{
Tiemo Landes, ${ }^{1,2, *}$ Markus Allgaier $\odot,{ }^{1}$ Sofiane Merkouche $\odot,{ }^{1}$ Brian J. Smith, ${ }^{1}$ \\ Andrew H. Marcus ${ }^{\circ},{ }^{2}$ and Michael G. Raymer (i) ${ }^{1}$ \\ ${ }^{1}$ Department of Physics and Oregon Center for Optical, Molecular, and Quantum Science, University of Oregon, Eugene, Oregon 97403, USA \\ ${ }^{2}$ Department of Chemistry and Biochemistry, Center for Optical, Molecular and Quantum Science, University of Oregon, \\ Eugene, Oregon 97403, USA
}

(Received 18 December 2020; accepted 28 June 2021; published 13 August 2021)

\begin{abstract}
Entangled photon pairs have promised to deliver a substantial quantum advantage for two-photon absorption spectroscopy. However, recent work has challenged the previously reported magnitude of quantum enhancement in two-photon absorption. We present a measurement of molecular absorption driven by isolated photon pairs using sum-frequency generation in a nonlinear optical crystal as a calibration device. We thereby establish an upper bound on the enhancement for entangled two-photon absorption in Rhodamine 6G, which lies well below previously reported values.
\end{abstract}

DOI: 10.1103/PhysRevResearch.3.033154

\section{INTRODUCTION}

Quantum states of light have been employed as a resource in disciplines from telecommunications to metrology to spectroscopy, with the goal of enhancing existing techniques with a quantum advantage. In the past decade, the use of time-frequency-entangled photon pairs promised enhanced two-photon absorption (TPA) for molecular and atomic spectroscopy, partly in an attempt to circumvent the strong optical powers required to observe TPA [1,2]. Several TPA experiments aimed at observing quantum enhancement using entangled photon pairs (EPPs) have been performed using a variety of molecular systems [3-8]. Recent publications have reported direct observation of entangled TPA-induced fluorescence [6-8], although with small yield. The reported strength of TPA with EPPs is within a few orders of magnitude of single-photon absorption, which translates to a quantum enhancement of more than 10 orders of magnitude when compared to TPA with nonentangled light. This enhancement has recently been challenged by new experimental results, suggesting that any enhancement is too small to be observed in realistic measurements, where expected signals are small to begin with [9].

Theoretically, many aspects of entangled two-photon absorption (ETPA) have been described, namely, the interaction timescales arising from time-frequency entanglement $[10,11]$. However, they are often neglected to account for realistic rates at which signal can be obtained in different regimes

\footnotetext{
*oqt@uoregon.edu

Published by the American Physical Society under the terms of the Creative Commons Attribution 4.0 International license. Further distribution of this work must maintain attribution to the author(s) and the published article's title, journal citation, and DOI.
}

with regards to flux, optical pulse duration, and the amount of time-frequency entanglement. Recent calculations cast doubt on previously published experimental results and thus prompt further investigation into the magnitude of TPA enhancement using EPPs [12-14]. That approach emphasizes the separate roles played by spectral correlations and by photon-number correlations. These new predictions suggest that large enhancement of the TPA cross section through time-frequency entanglement occurs only for narrow-band transitions, which are not typically present in molecular solutions. The advantage provided by photon-number correlations is predicted to be operable only in the limit of extremely low photon flux, where signals are practically unobservable below common detection thresholds. The quantum enhancement factor (QEF) for broadband transitions, taken as the ratio of TPA signal produced from EPPs and that generated from classical light with the same photon flux, depends only on EPP bandwidth $B$ and photon flux $F$ (both in hertz) and is given by QEF $\approx 2 \pi B / F$. While the enhancement can be several orders of magnitude, it is predicted to be insufficient for observing TPA-induced fluorescence at typical EPP flux [13]. These predictions are consistent with results of a series of ETPA experiments with a variety of dyes [9], in which a lack of observable TPA-induced fluorescence was used to bound the quantum enhancement. That survey makes several convincing arguments regarding the nature of the linear photon-flux scaling behavior that is often associated with ETPA. However, additional experimental steps are warranted in the absence of a fluorescence signal. Adverse effects such as optical dispersion, spatial overlap of photon pairs in the focal volume, and the precise photon-flux scaling behavior associated with two-photon processes and two-photon beams are all considered in our experiment.

In this paper, we present experimental results comparing sum-frequency generation (SFG) and molecular TPA in Rhodamine $6 \mathrm{G}$ using the same setup, each driven by isolated EPPs. SFG is known to be an excellent model for ETPA 
$[15,16]$. The results, consistent with recent theory $[12,13]$, provide a limit on the quantum advantage in molecular TPA. In our carefully controlled experiment the enhancement of TPA in Rhodamine 6G by EPPs is found to be no greater than several orders of magnitude below previously reported values. The upper bound we can place on the enhancement is no more than $1.5( \pm 0.3) \times 10^{5}$ times greater than what is predicted by the theory, putting TPA in the unobservable regime in this common test system.

Valuable test-bed systems for the study of the general nature of two-photon interaction have been TPA in atoms $[17,18]$ and SFG in second-order nonlinear crystals $[19,20]$. Timefrequency entanglement in SFG can be understood in terms of time-frequency modes [16,21]. TPA experiments on atoms revealed the importance of photon-number correlations. SFG serves to study the photon-flux scaling behavior of TPA when EPPs are employed. In molecular TPA, observing scaling behavior of absorption and fluorescence that is quadratic in power of an incident coherent-state beam is an essential piece of evidence for ruling out single-photon processes. We note that none of the observations of ETPA in molecular samples mentioned here [3-8] reported this rigorous scaling test.

The experiments presented here address three key elements that have yet to be convincingly discussed in prior work. Addressing these elements enables us to provide a more concrete bound on the potential quantum enhancement in molecular ETPA. First, in the low-flux regime, where theory predicts illumination by EPPs should produce a higher TPA signal compared with classical laser light, the crucial distinction lies in the scaling of the TPA signal arising from EPP. The TPA signal should scale linearly with the EPP pump power and quadratically when the EPP flux is directly attenuated, as shown for SFG [19]. A second condition necessary to provide convincing evidence of enhanced TPA is a large amount of time-frequency entanglement in the EPP state. Pumping spontaneous parametric downconversion (SPDC) with a spectrally narrow laser results in a high degree of spectral entanglement and temporal isolation of pairs. Therefore, a continuous-wave (cw) pumped SPDC source, as used in [8], is preferable over a pulse-pumped SPDC source for observing quantum enhancement of TPA, especially for generating large numbers of isolated pairs. Careful characterization of the interaction volume and EPP flux in that volume are essential for precisely bounding the quantum enhancement of TPA with EPPs. The dye employed here, Rhodamine $6 \mathrm{G}$, does not have intermediate states below the excited state, which would enhance TPA at the employed wavelength.

In what follows, we first describe the experimental setup and a study of TPA scaling behavior of SFG with flux for both EPPs and classical light. We then determine the expected TPA fluorescence signal from the molecular sample. A comparison with the detection noise threshold allows us to establish an upper bound on the enhancement of ETPA.

\section{EXPERIMENTAL SETUP}

The experimental setup sketched in Fig. 1 has two parts: light generation [Figs. 1(a) and 1(b)] and a detection block [Figs. 1(c)-1(e)]. A type-0 SPDC source, pumped by a 532-nm cw laser provides EPPs centered around $1064 \mathrm{~nm}$
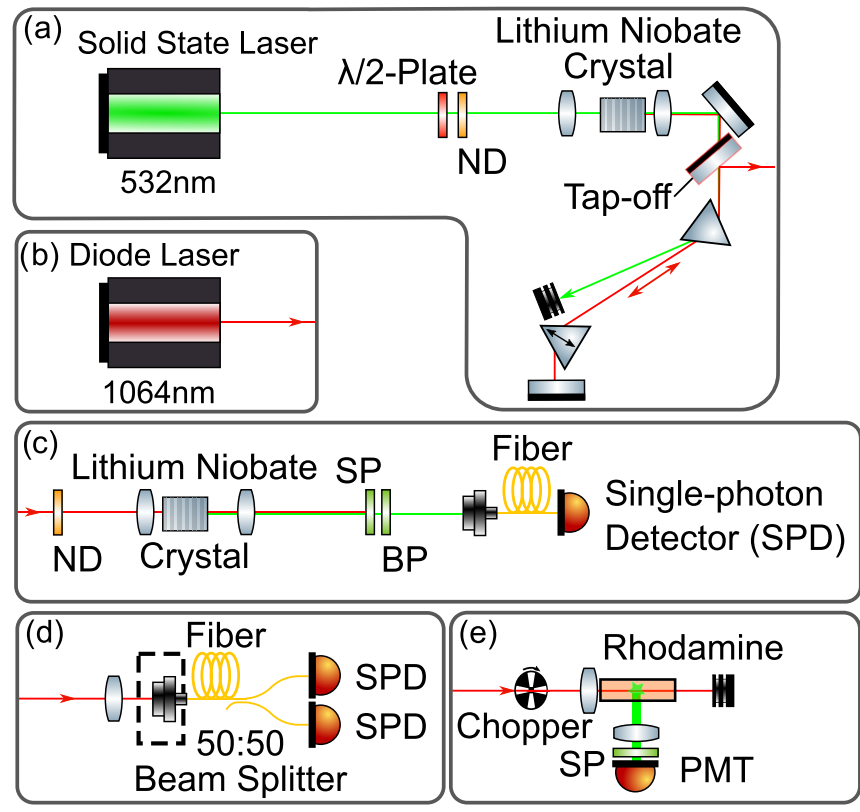

FIG. 1. For measurement of scaling behavior, either (a) the entangled photon pairs from a lithium niobate source or (b) coherent light from a diode laser are coupled through (c) another lithium niobate crystal. The SFG is then coupled into a single-mode fiber, which is connected to a single-photon detector. Neutral density (ND) filters in front and behind the EPP source are used to compare scaling behavior. Calibration of the fluorescence collection apparatus is performed by observing (e) the two-photon absorption from coherent light in (b). We then replace the molecular sample with (d) a singlemode fiber in the same place to obtain a lower bound on the number of photon pairs emitted by (a). PMT: large-area photo multiplier tube, SP: short-pass filter, BP: bandpass filter.

[Fig. 1(a)]. A prism pulse compressor is employed to compensate for second-order dispersion. Details on the EPP source and pulse compressor, which also removes the 532-nm pump light, are explained in the Appendix. Dispersion compensation is later verified by maximizing the signal from SFG [Fig. 1(c)]. Residual pump light is further blocked with several long-pass filters. As a calibration source we employ a cw diode laser at $1064 \mathrm{~nm}$ [Fig. 1(b)].

In the first experiment [Fig. 1(c)] we study SFG by isolated EPP to confirm that all requirements for successful ETPA, i.e., dispersion compensation, EPP collection, and focusing, are met. A collimated EPP emerging from the prism compressor is focused into an identical nonlinear crystal to perform SFG. Residual EPPs are removed using both short- and bandpass filters. Light generated via SFG is coupled into a single-mode fiber and detected on an avalanche photodiode. This experiment is repeated with the $1064-\mathrm{nm} \mathrm{cw}$ diode laser replacing the EPPs. The diode laser, SPDC, and SPDC pump are attenuated in order to verify scaling behavior of the SFG output counts with input IR power.

To examine the behavior of molecular ETPA, we first bound the EPP flux passing through the molecular sample. This binding is achieved by optimizing the two-photon flux collected by a single-mode fiber (SMF) placed at the beam focus within the sample cell. The fiber assembly is designed to be removed without misaligning the focusing lens or any prior 


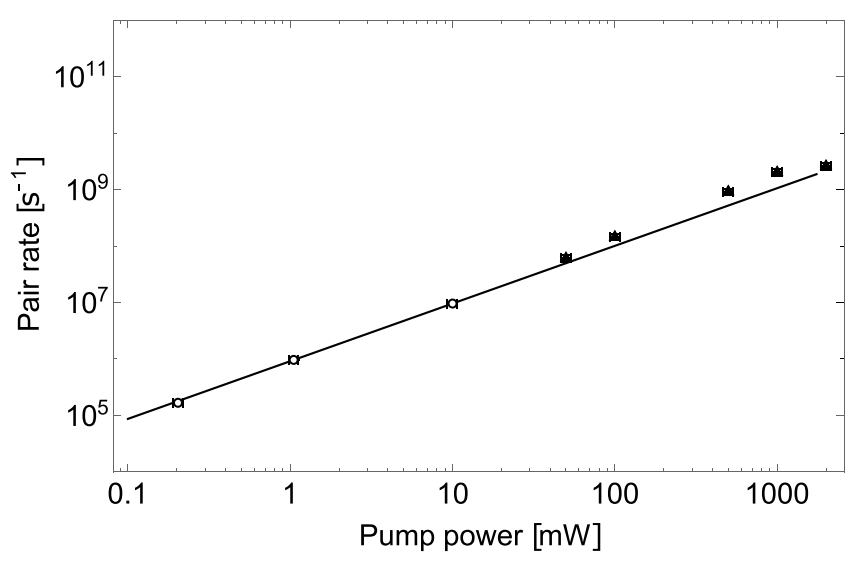

FIG. 2. Photon pair coincidence rate as a function of pump power. The measured rate is adjusted for the attenuation needed to prevent detector saturation (open circles). Each data point was averaged over $5 \mathrm{~s}$. The solid line represents an exponential fit to the linear regime (open circles); the exponent is $1.02 \pm 0.01$. Points at higher power were calculated using Klyshko efficiency in the linear regime and single count rates (solid triangles).

optics. The fiber-coupled EPPs are split probabilistically using a 50:50 fiber beam splitter and detected on superconducting nanowire single-photon detectors with a quantum efficiency of roughly $80 \%$ at $1064 \mathrm{~nm}$. The fiber provides a small test volume to make sure that generated pairs are tightly focused. Using a small test volume provides a much tighter bound on the delivered pair flux than simply measuring power or singlephoton counts in free space, especially in the presence of loss $L$, where the coincidence rate scales as $1 / L^{2}$. By design, only a fraction of EPPs are collected. While we cannot exclude higher-order spatial modes containing correlated pairs, coupling into a single-mode fiber provides a reliable lower bound on EPP flux.

We attenuate the EPPs to avoid detector saturation and measure the number of coincidences at low and medium flux. The adjusted number of counted pairs as a function of pump power is shown in Fig. 2. In the regime of isolated photon pairs the scaling is expected to be linear. We use those data points to calculate the Klyshko efficiency $\eta_{K}=16 \%( \pm 2 \%)$ and estimate the high-power coincidence rates by multiplying the single count rates with $\eta_{K}$ [22]. The pair rate estimated in this way is a conservative lower bound as it excludes any possible higher-photon-number contributions. This estimate is preferable to measuring optical power and making the assumption that there are exclusively correlated pairs in the beam, which is unrealistic in the presence of any loss. Obtaining a lower bound for the number of pairs in the described way allows us to account for any losses in the setup up to that point, such as reflection losses on metal mirrors, which would not be possible by inferring the pair rate from measured power at the SPDC wavelength. Accounting for losses is crucial since typical values for the Klyshko efficiency are on the order of $10 \%$. Without a small test volume such as an optical fiber, spatial overlap of counted pairs is never guaranteed. Any estimation of pair rates from total power is an overestimate by at least one or two orders of magnitude.

Above $1 \mathrm{~W}$ of SPDC pump power we observe a deviation from linear scaling, which we attribute to photorefraction in the SPDC crystal in the form of diminishing returns in pair production. We refrained from exceeding that power in our experiments. We measure $2.0 \times 10^{9}$ pairs/s at $1-\mathrm{W}$ pump power. At this rate, the average separation of two pairs is roughly $500 \mathrm{ps,}$, three orders of magnitude larger than their correlation time of $100 \mathrm{fs}$, as estimated from the measured EPP bandwidth.

With the EPP flux calibrated, the optical fiber is replaced with a 10 -mm-thick cuvette containing Rhodamine $6 \mathrm{G}$ in ethanol solution with a concentration of $2 \mathrm{mM}$ [Fig. 1(e)]. We find when illuminating with the IR laser that this concentration yields the strongest TPA-induced fluorescence signal in the present collection geometry. Details on the concentration dependence of yield and collection efficiency can be found in the Appendix. Fluorescence is collected on a large-area photomultiplier tube (Hamamatsu H7421-50) oriented at a $90^{\circ}$ angle from the incident light. Any scattered IR light is blocked using several short-pass filters.

For completeness, we compared the performance of this geometry with a more tightly focused backward-collection geometry imaged onto a photon-counting avalanche photodiode (see the Appendix). While the detector efficiency and signal-to-noise ratio improved in this setup, the collection efficiency was diminished, yielding only small net improvements to overall fluorescence detection. Despite improved detection, this geometry was unable to provide tighter bounds due to reduced coupling efficiency into the single-mode fiber.

\section{SCALING BEHAVIOR}

The first aspect of this investigation is the scaling of the generated signal with input flux for a well-understood twophoton process. For this test we employ an SFG process in a crystal that is identical to the SPDC crystal. While there can be spectral enhancement for SFG [23], we cannot quantify it in this experiment and merely use the SFG experiment to test the scaling behavior with photon flux. For this SFG experiment [Fig. 1(c)] we compare EPPs and classical cw light. The resulting SFG output count rate as a function of IR power, representing either the laser light or EPPs, is plotted on a log-log scale in Fig. 3. For comparison, we show lines corresponding to linear and quadratic scaling.

Observing quadratic scaling when varying EPP power directly by attenuation after the SPDC crystal verifies that we are, in fact, probing a two-photon process. Any single-photon process such as one-photon absorption or scattering would scale linearly. Thus, linear scaling with IR power alone is not a signature of ETPA. If, instead, the SPDC pump power is reduced, the number of produced EPPs is reduced while retaining photon-number correlations compared to probabilistically attenuating the photons individually, and scaling with flux is linear [19]. As long as optical power is far below a certain threshold, SFG driven by EPPs can provide efficiency orders of magnitude higher than classical cw light due to photon-number correlations.

Showing quadratic scaling with flux is essential for probing ETPA since the signature of TPA with its dependence on pair rate is easily mimicked by small parasitic single-photon processes. Given that for SFG we have obtained pure quadratic scaling with pair attenuation and pure linear scaling with 


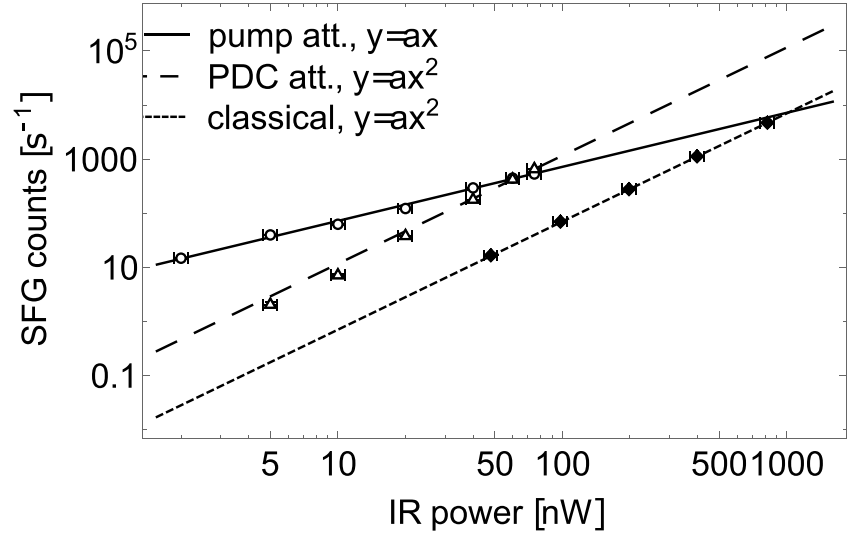

FIG. 3. SFG counts at $532 \mathrm{~nm}$ as a function of IR power for three cases: EPP where power is varied in the parametric down-conversion pump beam (open circles), EPP where power is varied after pair generation (open triangles), and a quasicoherent state from a diode laser (solid diamonds). We show functions corresponding to linear scaling (solid line) and quadratic scaling (dashed and dotted lines). Each data point was averaged over $180 \mathrm{~s}$ and had its dark count rate subtracted. Vertical and horizontal error bars represent shot noise and the measurement accuracy of the optical power meter, respectively.

pump attenuation, we can be confident that we are addressing two-photon processes. This is in line with past observations from $[17,19]$.

\section{TPA OF ENTANGLED PHOTON PAIRS}

Given we ascertained correct flux scaling behavior and dispersion compensation in SFG, we move on to studying the magnitude of the enhancement of TPA by EPPs. To obtain an upper bound analogous to that in [9], we need two numbers: the minimum supplied number of photon pairs in the interaction volume and the minimum detectable fluorescence count rate in our setup.

To obtain the usable EPP rate, we place the tip of a single-mode optical fiber (core diameter of $4 \mu \mathrm{m}$ ) in place of the rhodamine sample [compare Fig. 1(d)]. We focus with a 13-mm achromatic lens in order to achieve tight confinement and align the fiber to the lens. We use a 50:50 fiber beam splitter to split pairs probabilistically and detect photon pairs in coincidence at two superconducting nanowire single-photon detectors. Their efficiency is spectrally flat at $80 \%$ within a few percent around $1064 \mathrm{~nm}$. After correcting for known sources of loss, namely, detector efficiency and attenuation necessary to prevent detector saturation, we find the lower bound on the maximum usable pair rate in the fiber is $2.0( \pm 0.2) \times 10^{9} \mathrm{~s}^{-1}$, measured at a pump power of $1 \mathrm{~W}$. A complete description of the measurement can be found in the Appendix.

Next, we replace the fiber with a cuvette containing a $2 \mathrm{mM}$ solution of Rhodamine 6G. We found this concentration to yield maximum detected fluorescence from TPA. We excite a two-photon transition at $1064 \mathrm{~nm}$ with classical light from the cw IR laser and measure the TPA-induced fluorescence count rate. The results are shown in Fig. 4. The observed flux scaling agrees very well with the expected quadratic dependence. At the lowest power where we could observe a signal and after

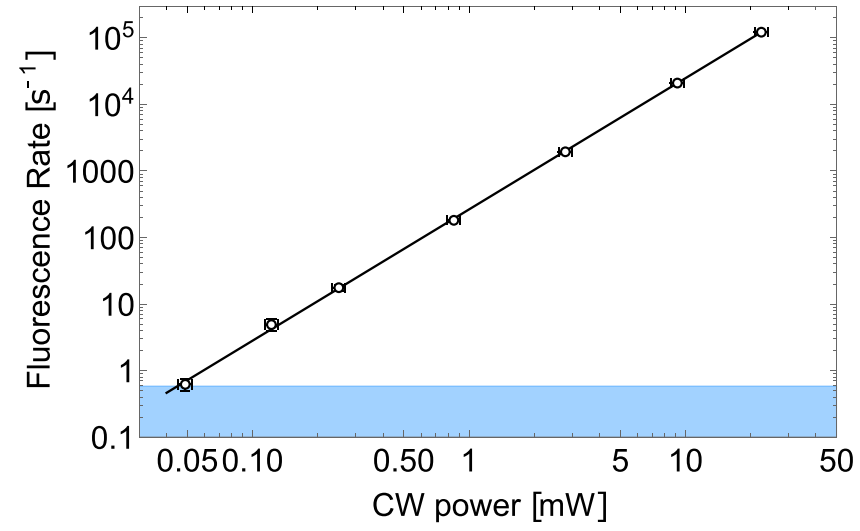

FIG. 4. Fluorescence count rates from laser-driven TPA in Rhodamine 6G. Counts rates were averaged over $5 \mathrm{~s}$ and for $1800 \mathrm{~s}$ for the lowest point. Horizontal error bars reflect the measurement accuracy of the optical power meter; vertical error bars represent shot noise. The fit exponent is $1.972 \pm 0.001$. The blue shaded area corresponds to fluorescence flux levels below the detection threshold.

subtracting dark counts, we measure a fluorescence rate of $0.7( \pm 0.1) \mathrm{s}^{-1}$, which we define as the detection threshold for this setup.

From these measurements we extract the collection efficiency $\eta_{\text {col }}$ by fitting the TPA fluorescence rates with the quadratic function TPA $=a F^{2}$ of the flux $F=P \lambda / h c$, where $P$ is optical IR power, $\lambda$ is the laser wavelength, $h$ is Planck's constant, and $c$ is the vacuum speed of light:

$$
\mathrm{TPA}_{\text {laser }}=\frac{F^{2} C \sigma_{2}}{\pi} \eta_{\mathrm{col}} \eta_{\text {det }} \gamma \int \frac{d z}{w(z)^{2}},
$$

where $C$ denotes concentration, $\sigma_{2}$ is the known conventional TPA cross section $9.4( \pm 1.5)$ GM [24], $\gamma=0.8$ is the known fluorescence yield, $\eta_{\text {det }}=10 \%$ is the known detection efficiency, and $w(z)$ is the beam waist along the optical axis. The obtained collection efficiency is $\eta_{\text {col }}=1.9 \%( \pm 0.2 \%)$, which is in reasonable agreement with independent estimates for our setup. This number enables us to estimate the expected fluorescence flux at the measured EPP rate. To account for the expected enhancement of photon-number correlations in EPPs relative to classical, uncorrelated $\mathrm{cw}$ light, we introduce a quantum enhancement factor, $\mathrm{QEF}=2 \pi B / F=$ $1.6( \pm 0.1) \times 10^{4}$, where $B$ denotes the measured spectral bandwidth of the EPP (see the Appendix for details):

$$
\begin{aligned}
\mathrm{TPA}_{\mathrm{EPP}} & =\mathrm{QEF} \frac{F^{2} C \sigma_{2}}{\pi} \eta_{\mathrm{col}} \eta_{\mathrm{det}} \gamma \int \frac{d z}{w(z)^{2}} \\
& =2.2( \pm 0.3) \times 10^{-6} \mathrm{~s}^{-1} .
\end{aligned}
$$

The ratio between the detection threshold and expected fluorescence rate bounds any additional quantum enhancement $E$ to the value

$$
E<\frac{0.7 \mathrm{~s}^{-1}}{2.2 \times 10^{-6} \mathrm{~s}^{-1}}=3.2( \pm 0.7) \times 10^{5} .
$$

This bound is prudent since the lower bound on the pair rate is conservative. Note that our model (see the Appendix for details) accounts for the positive effect of photon-number correlations. This additional quantum enhancement factor absorbs any additional enhancement, whether it stems from 
time-frequency entanglement or otherwise. This measured bound on $E$ is many orders of magnitude below reported experimental values, which range from $10^{10}$ [8] to $10^{31}$ [3] (extrapolated from published values for an "enhanced" cross section). While this bound would depend on EPP flux, the flux in our experiment is comparable to that in previous experiments. Any further decrease in flux would increase the theoretical enhancement but further reduce a signal that is already below practical detection thresholds.

From the measurements presented we conclude that any enhancement of two-photon absorption by time-frequencyentangled photon pairs is many orders of magnitude lower than previously reported. This finding supports the theoretical predictions in $[12,13]$ that quantum enhancement is equal to $2 \pi B / F$, the ratio of EPP bandwidth $B$ to flux $F$. For our experiment, carried out with $\mathrm{cw}$ sources where the enhancement should be maximal, this prediction yields $2 \pi B / F=$ $1.6( \pm 0.1) \times 10^{4}$, falling far short of enabling a successful detection of ETPA using state-of-the-art techniques. It also corroborates the independent results obtained under different controlled experimental conditions by Parzuchowski et al. [9]. While our experimental design can rule out some adverse factors that might diminish ETPA, namely, the lack of spatial overlap, dispersion, and dominating single-photon effects such as scattering, it should be recognized that these results are limited to the regime of isolated, nonoverlapping photon pairs and that these findings apply only to molecular dyes with broadband absorption spectra. A discussion of other possible prohibitive effects in observing fluorescence is included in the Appendix. Using nonlinear crystals with higher power tolerance for EPP generation would allow us to reach higher pair rates but would also prompt us to leave the regime of isolated pairs, which violates the conditions required for creating a large quantum enhancement.

These results further emphasize the need for careful characterization and validation of ETPA experiments, such as fluorescence detection and verification of quadratic scaling of the two-photon interaction signal with attenuation of the entangled photons after the SPDC crystal, as demonstrated with SFG in this work and in [19]. Such experiments rule out spurious signals that could mimic the linear scaling of ETPA such as single-photon absorption from molecular aggregates or other impurities, linear scattering processes, fluorescence from optical elements, and residual SPDC-pump breakthrough. These experiments would also provide valuable insights into the nature of the processes being observed, such as enhancement beyond what is predicted in [12,13], which could stem from squeezing, spatial correlations, or novel features in the molecular electronic structure.

\section{ACKNOWLEDGMENTS}

This work was supported by the National Science Foundation RAISE-TAQS Program (Grant No. PHY-1839216 to M.G.R., A.H.M., and B.J.S. as co-principal investigators).

\section{APPENDIX}

We first give experimental details of the SPDC source and dispersion compensator, then discuss expected TPA rates,

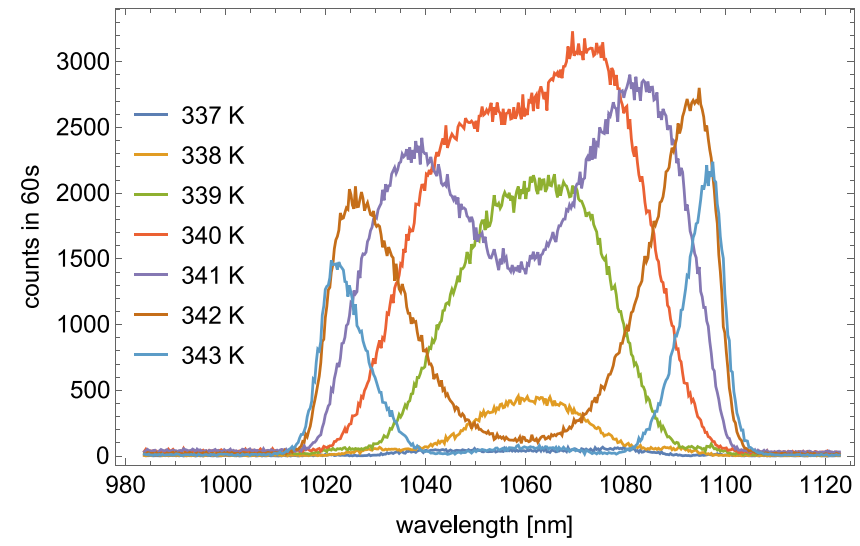

FIG. 5. Spectrum of the entangled photon pairs at various crystal temperatures. Spectra were measured on a 500-m-long dispersive fiber time-of-flight spectrometer.

the effects of varying molecular concentration and collection geometry, and, finally, several other potential adverse effects.

\section{Source parameters and spectrum}

The SPDC source uses a periodically poled, 10-mm-long magnesium oxide-doped lithium niobate bulk crystal (Covesion) with a poling period of $6.90 \mu \mathrm{m}$ and a phase-matching temperature of $340 \mathrm{~K}$. The type- 0 process is pumped with $\mathrm{cw}$ light from a diode-pumped solid-state laser at $532 \mathrm{~nm}$ (Coherent Verdi V-5). The forward-propagating (colinear) part of the SPDC mode is collimated with an achromatic lens with a focal length of $100 \mathrm{~mm}$. The spatial beam properties of the SPDC, including collimation and copropagation with the 1064-nm laser beam, are verified using a CCD camera at various distances from the collection lens.

The emitted SPDC, centered around $1064 \mathrm{~nm}$, was sent through $1000 \mathrm{~m}$ of optical fiber (Nufern 780HP), facilitating a fiber-assisted time-of-flight spectrometer analogous to that in [25].

Figure 5 shows measured EPP spectra. They include both signal and idler photons and thus present the sum of signal and idler marginal spectra. The FWHM bandwidth extracted from a Gaussian fit is $40 \mathrm{~nm}(10.6 \mathrm{THz})$. Since the shape of a cw-pumped type-0 PDC is well known, we can estimate the number of temporal modes $K \approx \sqrt{2} \Delta v_{p d c} / \Delta v_{\text {pump }}$ from the pump beam linewidth. The pump laser (Coherent Verdi V-5) linewidth is $5 \mathrm{MHz}(\mathrm{rms})$ or $6.8 \mathrm{MHz}(\mathrm{FWHM})$ :

$$
K=\sqrt{2} \times 10.6 \mathrm{THz} / 6.8 \mathrm{MHz}=2.2 \times 10^{6} .
$$

\section{Dispersion compensation}

Because of the broadband EPP spectrum, dispersion plays an important role in this experiment. Even small amounts of dispersion in the PDC crystal, lenses, and filters, which amount to roughly $4000 \mathrm{fs}^{2}$, cause the photons within each pair to lose temporal overlap. To avoid this, we compensate second-order dispersion in a fashion analog to the $\chi^{2}$ experiments conducted by Dayan et al. [19]. Figure 6 shows a more detailed schematic of the employed prism compressor. 


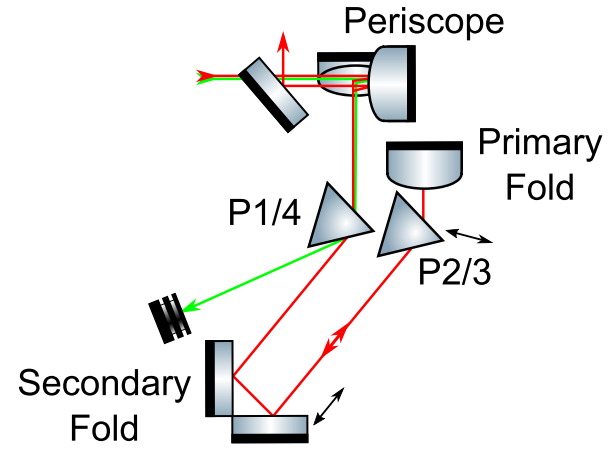

FIG. 6. Schematic of the prism compressor. P1, P2, P3, P4: prisms in the order the beam passes through them.

In considering the wavelength, possibility for alignment, and amount of expected dispersion, we chose to use a doublefolded prism compressor with SF11 prisms. For zero prism insertion the prism separation would be approximately $20 \mathrm{~cm}$. The amount of positive dispersion applied with $10 \mathrm{~mm}$ of prism dispersion would roughly cancel out the negative dispersion achieved by the compressor, allowing for a large degree of variability while guaranteeing stable beam alignment.

To enter the prism compressor and take advantage of the lack of reflection at the Brewster angle, polarization has to be rotated. Due to the broadband spectrum of the EPP we do this with a periscope.

The prism compressor, consisting of four effective prisms, is folded in two places: between prisms 2 and 3 (main fold), which enables us to use the prism in a double pass, and between prisms 1 and 2 (secondary fold), which are effectively also prisms 3 and 4 , which enables us to change prism separation without realignment. Each fold uses a retroreflector (roof mirror or two mirrors oriented at $90^{\circ}$ to each other). The main fold uses a vertical roof mirror. It allows us to keep the incoming and outgoing beams parallel to each other and to the prism axis but at a different height from the table. This beam geometry eliminates any asymmetry in the beam mode. The secondary fold uses a horizontal roof mirror on a precision ball-bearing translation rail. The green pump beam does not hit the second prism and is dumped. The beam of the 1064-nm diode laser is used for initial alignment. Both prisms sit on turntables and are aligned to minimum deviation using the diode. After alignment, we verified on a CCD camera that the entire PDC beam mode passes the prism compressor and periscope and is unobstructed. We also verified with the camera in several positions that there is no significant beam walk-off due to alignment. An advantage of folding the prism compressor between prisms 2 and 3 is that shift caused by changing the insertion of those prisms is inherently canceled out. This serves as an important tool to verify that changes in signal intensity are caused by dispersion rather than misalignment. We can observe this invariance directly when we measure the number of pairs in a single-mode fiber and find that number to be insensitive to prism insertion.

The prism compressor is then finally used to maximize the signal obtained from SFG in the second crystal. SFG is the strongest at minimal dispersion. We adjust it by changing the prism separation using the retroreflector at the secondary fold. Around the maximum efficiency, we verify that changing prism separation and changing insertion of the second prism (which is inherently stable in terms of alignment) give the same result. We then lock prism separation and insertion in that position before performing all the experiments described in the main text.

To account for dispersion from the SFG crystal, we have also varied the compressor setting for the ETPA experiment, with no change in its outcome.

\section{Expected TPA fluorescence rate from EPPs}

To calculate the expected TPA fluorescence flux for excitation with EPPs, we use the established theory of classical TPA with monochromatic light, which relates fluorescence flux to the excitation flux $F$ :

$$
R_{T P A}=\frac{F^{2} C \sigma_{2}}{\pi} \eta_{\mathrm{col}} \eta_{\mathrm{det}} \gamma \int \frac{1}{w(z)^{2}},
$$

where $C$ denotes concentration (in molecules/volume), $\sigma_{2}$ is the classical TPA cross section, $\eta_{\text {col }}$ is the collection efficiency, $\eta_{\text {det }}$ is the detector efficiency, $\gamma$ denotes the fluorescence yield, and $w(z)$ is the beam waist along the optical axis. To adapt this relationship for positive effects of EPPs, we employ the definition of a quantum enhancement factor (QEF) from [13]

$$
\mathrm{QEF}=\frac{P_{f}^{E P P}}{P_{f}^{\mathrm{coh}}},
$$

where $P_{f}^{E P P}$ and $P_{f}^{\text {coh }}$ denote the probability of the respective states to excite the two-photon transition in a time $T$. For pulsed light, this interval will be the pulse duration. Here, we model cw light as being "chopped" into a series of square pulses of arbitrary duration $T$, which is defined in a way that forces $P \ll 1$.

For $\mathrm{cw}$ excitation, where the two-photon transition bandwidth is larger than the bandwidth of the exciting light, the TPA probability for a single molecule can be expressed as a function quadratic in the flux $F$ :

$$
P_{f}^{\mathrm{coh}}=\left(\frac{F}{A_{0}}\right)^{2} \sigma_{2} T .
$$

However, for EPPs the probability scales linearly with the photon pair flux (which is half the single rate):

$$
P_{f}^{E P P}=\left(\frac{F}{A_{0}}\right)\left(\frac{\sigma_{2}}{A_{0}}\right) 2 \pi B \times f_{E P P} T,
$$

where $B$ is the bandwidth of the EPPs in hertz and $f_{E P P}$ is a unitless factor described in [13]. For tightly focused, highly entangled states, as in this experiment, $f_{E P P}$ is approximately 1. The ratio of $P_{f}^{E P P}$ to $P_{f}^{\text {coh }}$ at equal flux then describes the ratio of encountering a pair per unit time interval:

$$
\mathrm{QEF}=\frac{2 \pi B}{F} .
$$

For the comparison this allows us to calculate the expected fluorescence flux including the positive effect of the EPP photon-number correlation. For a EPP with FWHM bandwidth of $40 \mathrm{~nm}(10.6 \mathrm{THz})$ and a flux of $4.0 \times 10^{9}$ the predicted enhancement factor is approximately $16000 \pm$ 1000. For a more intuitive picture, consider that the EPP 


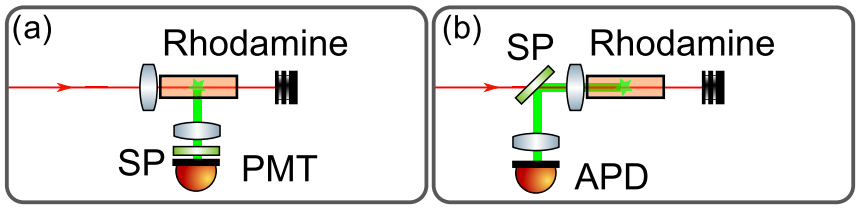

FIG. 7. Schematic of (a) the $90^{\circ}$ collection geometry and (b) back-reflection geometry. PMT: photomultiplier tube, SP: shortpass filter, APD: avalanche photodiode.

enhancement is the inverse of the probability to encounter a pair of uncorrelated (classical) photons within the correlation time of the EPP. The lower the photon rate is, the smaller this probability is, and the larger the relative enhancement is.

\section{Collection geometry}

We compared two different collection geometries in order to find one efficient and largely insensitive to alignment. A geometry where the detector is placed at $90^{\circ}$ from the incoming beam path allows us to collect fluorescence from the entire sample volume onto a large area photomultiplier tube [PMT; see Fig. 7(a)]. Due to it being fairly insensitive to alignment, this collection geometry was chosen for the main experiment since it decreases the chance of missing any entangled twophoton induced fluorescence. The focusing geometry into the sample also made it easy to switch between the sample and the calibration fiber, and reasonable fiber coupling efficiency was achieved.

A backward collection geometry was compared to the side collection geometry [see Fig. 7(b)]. The advantage is that imaging the fluorescence onto a smaller-area detector is easy since the backward fluorescence beam is automatically collimated through the focusing lens. Therefore, a smaller-area single-photon counting avalanche photodiode (APD) can be used, which is superior in signal-to-noise in comparison to the PMT. However, we found that collection efficiency was decreased and no significant gain in signal collection could be achieved compared to the side collection geometry, while backward collection was highly sensitive to alignment. In addition, fiber coupling efficiency was poor. The main advantage of this geometry is that fluorescence emitted close to the facet of the cuvette can be detected, even in the presence of strong fluorescence reabsorption that would otherwise prevent detection on the side. Nevertheless, the backwards geometry proved useful for studying concentration effects.

\section{Concentration dependence}

At low concentrations the rate of TPA and collected fluorescence scales linearly with the concentration. At very high concentrations, however, various effects reduce the fluorescence rate. Fluorescence quenching due to particle aggregation and fluorescence reabsorption are two such effects that place practical limitations on the concentration that can be utilized for efficient fluorescence collection.

These effects were investigated in the backwards collection geometry using a short-focal-length aspheric lens $(3 \mathrm{~mm})$ and a free space APD. The 3-mm lens serves as both the focusing lens for the incident beam and the collection optic for the
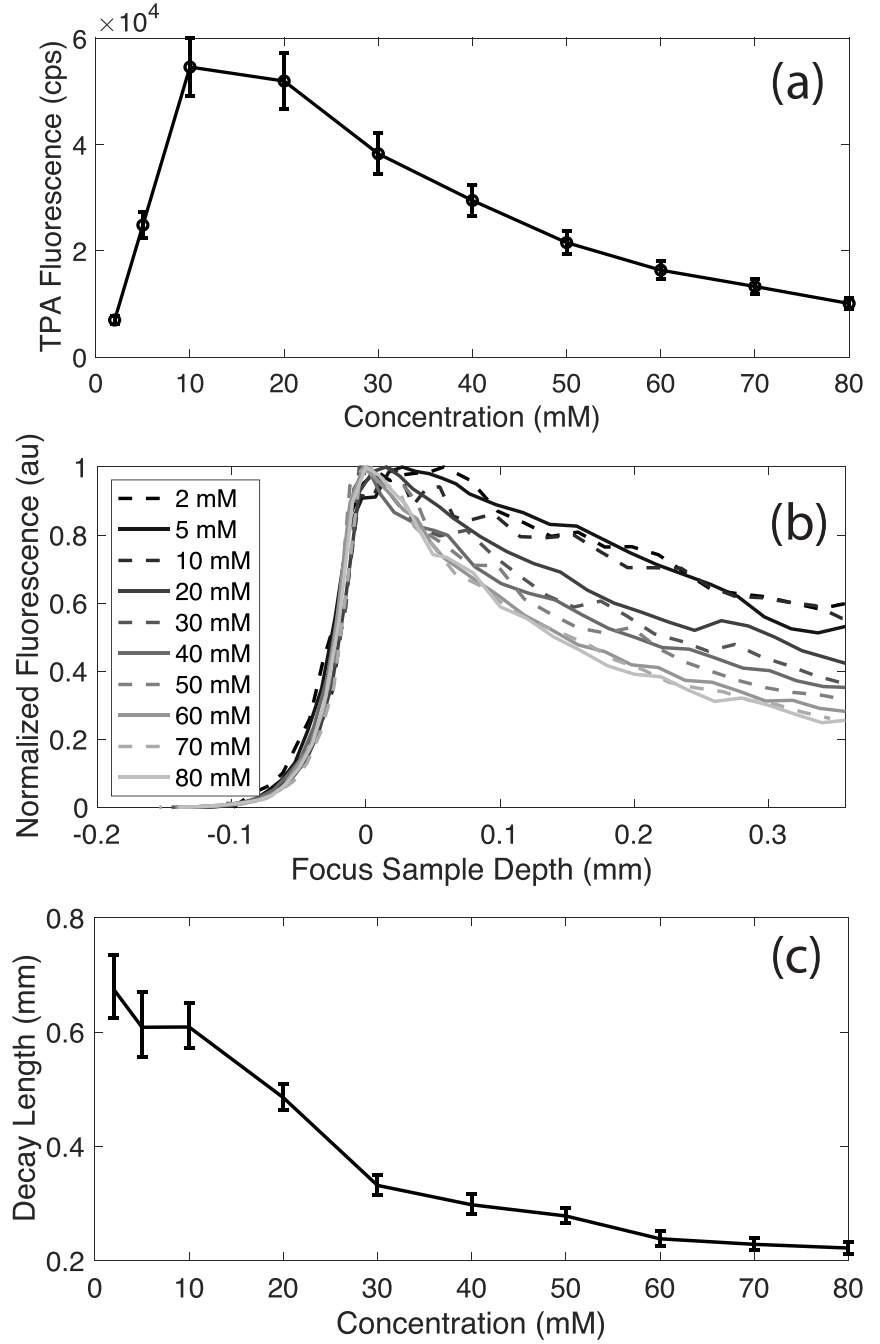

FIG. 8. Plots of (a) maximum collected TPA fluorescence as a function of concentration, (b) normalized TPA fluorescence intensity as a function of focal position relative to the front face of the cuvette, and (c) decay of fluorescence intensity with focal position, as quantified by a fit to an exponential decay $e^{-x / L}$, where $x$ is the distance into the sample, shown with $95 \%$ confidence intervals. All measurements are at $10-\mathrm{mW} \mathrm{cw}$ laser excitation.

detection. The collimated TPA fluorescence is reflected off a dichroic mirror and focused onto a free space APD.

This configuration reduces the effect of reabsorption by collecting at the front face of the sample volume and allows collection at concentrations limited mainly by aggregation quenching effects. Despite this benefit the total collected TPA declines past a concentration of $20 \mathrm{mM}$, as seen in Fig. 8(a). Figure 8(b) shows the normalized TPA fluorescence collected as the sample is moved closer to the focusing lens. After a sharp increase, corresponding to the front face of the sample overlapping the focal point, the collection efficiency begins to decrease. While effects such as increasing optical aberrations with focus depth can contribute to this effect, the concentration dependence seen in Fig. 8(c) suggests reabsorption or pump depletion in the Rhodamine 6G solution. (Although pump depletion from TPA alone is negligible, other effects such as scatter or absorption may contribute to pump deple- 
tion.) Due to increased reabsorption in the side collection geometry, $2 \mathrm{mM}$ was close to optimal for that experiment. These results also indicate that while further optimization in sample concentration and geometry could be achieved, these effects do not represent orders of magnitude increases in sensitivity.

\section{Discussion of adverse effects}

Performing an experiment in the absence of a detectable signal is prone to many pitfalls. Here, we attempt to compile a comprehensive list of such pitfalls, along with our attempts to anticipate them. This list is in part compiled from Refs. [9,13] and amended with our own analysis of SPDC experiments.

Insufficient spatial overlap. If focusing into the molecular sample is insufficiently tight or the waist is not inside the sample volume, there is a chance that the two photons within a pair will have poor spatial overlap. We address this by using a single-mode fiber as a test volume for our actual photon flux. The pair rate is directly measured in coincidence.

Dispersion. Dispersion would cause the anticorrelated photons in a pair to arrive at different times and lose temporal overlap, preventing TPA, as quantified in [14]. We estimate the amount of dispersion from the specifications of all employed optics. This estimation allows us to design an appropriate prism compressor that compensates for second-order dispersion. We use the SFG experiment to verify successful dispersion compensation. The SMF experiment allows us to exclude any adverse effects from alignment caused by adjusting the amount of compression.

Competing single-photon processes. Scattering, the pump light bleeding through optical filters, single-photon absorption in the dye, solvents, optics, and the cuvette, to name just a few, all share the same linear scaling behavior as a function of optical loss. A linear scaling signature alone is therefore not sufficient to verify that TPA is the dominant process. It is therefore recommended verifying that TPA and fluorescence rates scale quadratically with direct attenuation of pairs, as demonstrated in our SFG experiment. A mixture of single- and two-photon processes would decrease the detection signal-to-noise ratio, and at least for classical TPA the proper scaling should be verified.

Linear loss. The effect of linear loss on photon pairs is that either photon can be lost probabilistically. Because of this, one cannot infer the average number of pairs from the average number of photons, or optical power. It is therefore necessary to directly measure the number of incident pairs in coincidence. Such a direct pair measurement allows us to ignore any sources of loss between the SPDC source and the molecular sample since the number of photon pairs is known at the sample location.

Detector saturation. Any and all of the signals one may wish to observe here rely on a linear detector response. As the average duration between photon pairs approaches the dead time of the detector, photons arriving in fast succession are undercounted, and detector response to power becomes sublinear. Dead time of common photon counting detectors is of the order of $50 \mathrm{~ns}$ for avalanche photodiodes, $10 \mathrm{~ns}$ for photomultiplier tubes, and as low as a few nanoseconds for superconducting nanowire detectors. Therefore, saturation effects usually start to appear at counts in excess of $10^{7} \mathrm{~s}^{-1}$. We attenuate the SPDC beam in order to avoid saturation and then adjust the measured coincidence rate for the amount of attenuation. At the onset of saturation, correction of the detector response may be possible with manufacturer-supplied calibration factors, but only for cw light. For pulsed light with an average photon number per pulse larger than 1 , detector dead time correction cannot yield reliable results.

Insufficient collection efficiency. The simplest explanation for the absence of a fluorescence signal is insufficient collection efficiency, but collection efficiency is easily characterized with classical TPA.

Asymmetric spectral detector response. Silicon-based detectors do not just suffer from low quantum efficiency near the optical band gap of $1100 \mathrm{~nm}$ but also have highly asymmetric spectral detection efficiencies, which biases detection around $1064 \mathrm{~nm}$ towards the short-wavelength half of the SPDC spectrum. Because of this, optimizing solely on counts can result in poor coupling at the center of the spectrum, so using raw single-photon count rates to align fiber coupling is not an option. There are three remedies: using a narrow-band spectral filter to align on the center of the spectrum, aligning directly on coincidences using a fiber beam splitter and two detectors, or using a different sort of detector with a more uniform response. The latter is the case when using nanowire detectors: In the range of 1000-1100 nm, their quantum efficiency varies by only a few perfect.

Insufficient EPP generation efficiency. SPDC sources rarely supply all of the flux in the form of pairs. The extent to which flux is in the form of pairs is typically expressed in the form of Klyshko efficiency [22], which is the ratio of the coincidence count rate to the single-photon count rate. The Klyshko efficiency quantifies how many usable pairs are contained in a beam emitted from an SPDC source and collected for detection (and experimentation). When the value of the Klyshko efficiency is corrected for detection efficiency, usually referred to as the heralding efficiency, it is typically of the order of $10 \%$ to $80 \%$ [26,27], depending on detection efficiency. The fact that only a fraction of photons in an SPDC beam are actually in pairs is another reason to optimize a setup on coincidence counts and measure the number of pairs rather than to assume them from power or single counts.

Reabsorption. The emission and absorption spectra of dyes such as rhodamine overlap, causing some of the emitted fluorescence photons to be reabsorbed. Higher dye concentration would increase the number of TPA events but would also increase reabsorption of fluorescence photons. For the side collection apparatus, using the fluorescence signal from classical TPA, we established that within the range of 0.2 to $20 \mathrm{mM}$, a concentration of $2 \mathrm{mM}$ maximized detection for our collection geometry without appreciable reabsorption.
[1] K. E. Dorfman, F. Schlawin, and S. Mukamel, Nonlinear optical signals and spectroscopy with quantum light, Rev. Mod. Phys. 88, 045008 (2016).
[2] S. Szoke, H. Liu, B. P. Hickam, M. He, and S. K. Cushing, Entangled light-matter interactions and spectroscopy, J. Mater. Chem. C 8, 10732 (2020). 
[3] D.-I. Lee and T. Goodson, Entangled photon absorption in an organic porphyrin dendrimer, J. Phys. Chem. B 110, 25582 (2006).

[4] J. P. Villabona-Monsalve, O. Calderón-Losada, M. Nuñez Portela, and A. Valencia, Entangled two photon absorption cross section on the $808 \mathrm{~nm}$ region for the common dyes zinc tetraphenylporphyrin and rhodamine b, J. Phys. Chem. A 121, 7869 (2017).

[5] A. Mikhaylov, K. M. Parzuchowski, M. D. Mazurek, D. J. Lum, T. Gerrits, C. H. Camp, Jr., M. J. Stevens, and R. Jimenez, A comprehensive experimental system for measuring molecular two-photon absorption using an ultrafast entangled photon pair excitation source, Proc. SPIE 11295, 48 (2020).

[6] O. Varnavski, B. Pinsky, and T. Goodson, Entangled photon excited fluorescence in organic materials: An ultrafast coincidence detector, J. Phys. Chem. Lett. 8, 388 (2017).

[7] J. P. Villabona-Monsalve, O. Varnavski, B. A. Palfey, and T. Goodson, Two-photon excitation of flavins and flavoproteins with classical and quantum light, J. Am. Chem. Soc. 140, 14562 (2018).

[8] D. Tabakaev, M. Montagnese, G. Haack, L. Bonacina, J.-P. Wolf, H. Zbinden, and R. T. Thew, Energy-time-entangled twophoton molecular absorption, Phys. Rev. A 103, 033701 (2021).

[9] K. M. Parzuchowski, A. Mikhaylov, M. D. Mazurek, R. N. Wilson, D. J. Lum, T. Gerrits, C. H. Camp, M. J. Stevens, and R. Jimenez, Setting Bounds on Entangled Two-Photon Absorption Cross Sections in Common Fluorophores, Phys. Rev. Appl. 15, 044012 (2021).

[10] H.-B. Fei, B. M. Jost, S. Popescu, B. E. A. Saleh, and M. C. Teich, Entanglement-Induced Two-Photon Transparency, Phys. Rev. Lett. 78, 1679 (1997).

[11] F. Schlawin, Entangled photon spectroscopy, J. Phys. B 50, 203001 (2017).

[12] M. G. Raymer, T. Landes, M. Allgaier, S. Merkouche, B. J. Smith, and A. H. Marcus, How large is the quantum enhancement of two-photon absorption by time-frequency entanglement of photon pairs?, Optica 8, 757 (2021).

[13] T. Landes, M. G. Raymer, M. Allgaier, S. Merkouche, B. J. Smith, and A. H. Marcus, Quantifying the enhancement of two-photon absorption due to spectral-temporal entanglement, Opt. Express 29, 20022 (2021).
[14] M. G. Raymer, T. Landes, and A. H. Marcus, Entangled twophoton absorption by atoms and molecules: A quantum optics tutorial, arXiv:2103.02551.

[15] B. Dayan, Theory of two-photon interactions with broadband down-converted light and entangled photons, Phys. Rev. A 76, 043813 (2007).

[16] S. Merkouche, V. Thiel, and B. J. Smith, Positive operatorvalued measure for two-photon detection via sum-frequency generation, Phys. Rev. A 103, 043711 (2021).

[17] N. Ph. Georgiades, E. S. Polzik, K. Edamatsu, H. J. Kimble, and A. S. Parkins, Nonclassical Excitation for Atoms in a Squeezed Vacuum, Phys. Rev. Lett. 75, 3426 (1995).

[18] B. Dayan, A. Pe'er, A. A. Friesem, and Y. Silberberg, Two Photon Absorption and Coherent Control with Broadband Down-Converted Light, Phys. Rev. Lett. 93, 023005 (2004).

[19] B. Dayan, A. Pe'er, A. A. Friesem, and Y. Silberberg, Nonlinear Interactions with an Ultrahigh Flux of Broadband Entangled Photons, Phys. Rev. Lett. 94, 043602 (2005).

[20] K. A. O'Donnell and V. G. Garces, Watching a photon interfere with itself, Opt. Commun. 463, 125441 (2020).

[21] C. Fabre and N. Treps, Modes and states in quantum optics, Rev. Mod. Phys. 92, 035005 (2020).

[22] D. N. Klyshko, Use of two-photon light for absolute calibration of photoelectric detectors, Sov. J. Quantum Electron. 10, 1112 (1980).

[23] A. Pe'er, B. Dayan, A. A. Friesem, and Y. Silberberg, Temporal Shaping of Entangled Photons, Phys. Rev. Lett. 94, 073601 (2005).

[24] N. S. Makarov, M. Drobizhev, and A. Rebane, Two-photon absorption standards in the 550-1600 nm excitation wavelength range, Opt. Express 16, 4029 (2008).

[25] M. Avenhaus, A. Eckstein, P. J. Mosley, and C. Silberhorn, Fiber-assisted single-photon spectrograph, Opt. Lett. 34, 2873 (2009).

[26] G. Harder, V. Ansari, B. Brecht, T. Dirmeier, C. Marquardt, and C. Silberhorn, An optimized photon pair source for quantum circuits, Opt. Express 21, 13975 (2013).

[27] M. Bock, A. Lenhard, C. Chunnilall, and C. Becher, Highly efficient heralded single-photon source for telecom wavelengths based on a ppln waveguide, Opt. Express 24, 23992 (2016). 